\title{
Generalized Auditory Same-Different Discrimination by Pigeons
}

\author{
Robert G. Cook and Daniel I. Brooks \\ Tufts University
}

\begin{abstract}
Three pigeons were trained in a successive same/different (S/D) procedure using compound auditory stimuli (pitch/timbre combinations). Using a go/no-go procedure, pigeons successfully learned to discriminate between sequences of either all same (AAAA . . . or BBBB ...) or all different (ABCD ...) sequences consisting of 12 sounds. Both pitch and timbre were subsequently established as controlling dimensions. Transfer tests with novel stimuli revealed a generalized basis for the discrimination (novel pitch/timbre combinations, novel pitches, novel instruments, and complex natural \& man-made sounds). These results indicate for the first time that pigeons can learn generalized same/different discriminations in a nondominant modality. When combined with earlier visual results, they support a qualitative similarity among birds and primates in their capacity to judge this type of fundamental stimulus relation across different modalities.
\end{abstract}

Keywords: audition, relational discrimination, same-different discrimination, pigeon, birds

The capacity to abstract and recognize generalized regularities and patterns is a critical cognitive component of intelligence. These abstractions serve the crucial function of predicting future events and have been an important focal point in the comparative study of learning, categorization, and intelligence. Whether animals can conceptualize the regularities associated with same and different relations among sets of elements has recently received a great deal of attention. In an S/D task, the subject responds "same" when two or more stimuli are identical and "different" if one or more of the stimuli are different from one another. The degree to which this learned behavior transfers to same and different relations involving novel elements is generally taken as evidence of concept formation. Among animals, nonhuman primates have regularly been shown to learn such relations (Thompson \& Oden, 2000; Wright, Cook, \& Kendrick, 1989; Wright, Santiago, \& Sands, 1984). Over the past 10 years, the idea that birds, as primarily tested with pigeons, can also visually discriminate same/ different and matching relations has also received repeated support (Cook, Katz, \& Cavoto, 1997; Cook, Kelly, \& Katz, 2003; Cook \& Wasserman, 2006; Katz \& Wright, 2006; Pepperberg, 1987; Young \& Wasserman, 2001).

So far, this comparative research has exclusively concentrated on the dominant sensory modality of each species. For example, all of the pigeon concept research has used only visual stimulation. Thus, it is unknown if this important cognitive capacity is modality specific or not. Here, we report for the first time that pigeons can discriminate and conceptualize S/D relations within their nondominant auditory modality.

Robert G. Cook and Daniel I. Brooks, Department of Psychology, Tufts University.

This research was supported by grant \#0316016 from the National Science Foundation.

Correspondence concerning this article should be addressed to Dr. Robert G. Cook, Department of Psychology, Tufts University, Medford, MA 02155. E-mail: Robert.Cook@tufts.edu
Demonstrating this cognitive capacity in a completely different modality is important for several reasons. First, it indicates that $\mathrm{S} / \mathrm{D}$ discrimination is a generalized competence common to different modalities in birds. Second, it raises the possibility that this capacity is potentially due to a single, higher-order abstraction mechanism that detects relations across different types of information and temporal arrangements (simultaneous \& successive methods of presentation). Such a capacity would be an important prerequisite in the evolution of acoustic communication and language. Third, it rules out broad classes of modality-specific perceptual hypotheses proposed as alternative accounts of the earlier visual S/D findings (Mackintosh, 2000).

While psychophysical information on pigeon audition has been established (Harrison \& Furumoto, 1971; Heise, 1953; Jenkins \& Harrison, 1960; Sinnott, Sachs, \& Hienz, 1980), little research has investigated advanced cognitive functions within this modality outside of short-term memory and event duration (Kraemer \& Roberts, 1985; Santi, Stanford, \& Coyle, 1998). This may be due to the fact that pigeons seem to have a bias to use visual cues over auditory cues in appetitive operant settings (Foree \& Lolordo, 1973; Kraemer \& Roberts, 1985) and an informal laboratory reputation of being difficult to train with acoustic stimuli. Nevertheless, while pigeons generally have a smaller and innate vocal repertoire in comparison to their more widely studied passerine and psittacine relatives, these vocalizations are an important facet of social control and reproductive behavior in this family of birds (Skutch, 1991).

The pigeons were tested using a go/no-go discrimination with sequences of 12 auditory stimuli that were either all the same (AAAA ...) or all different (ABCD ... .). The training stimuli were redundant compounds made from notes from two separate octaves (pitch) as played by different synthesized musical instruments (timbre). The pigeons pecked at a touch panel located between opposing speakers and were reinforced for pecking during the different sequences, but not same sequences. After acquiring the task, we examined the generalized nature of this auditory S/D 
discrimination by conducting six transfer tests using novel acoustic stimuli and properties.

\section{Method}

\section{Animals}

Three male pigeons (Columba livia) were tested. One (\#2N; 6-years old) had experience in a successive visual S/D task (Cook, Kelly, \& Katz, 2003). The other two were naïve (each 2-years old) They were maintained at $80-85 \%$ of their free-feeding weights in a 12:12 LD colony room.

\section{Apparatus}

Testing was done in a flat-black Plexiglas chamber $(42.5 \mathrm{~cm}$ wide $\times 44 \mathrm{~cm}$ deep $\times 39.5 \mathrm{~cm}$ high). All events were controlled by a computer with sound card (SoundMax Integrated audio card; Analog Devices). The visual stimuli were presented on a color monitor visible through a $25.5 \mathrm{~cm} \times 21.5 \mathrm{~cm}$ opening in the chamber's front panel. Pecks were detected by an infrared touch screen (EloTouch) that formed this window. The auditory stimuli were simultaneously presented from two multimedia speakers (HK-195, Harmon-Kardon) located toward the front of each side of the chamber. The speakers were played through plastic grills in the side of the chamber consisting of a $90 \times 120 \mathrm{~cm}$ grid of $5 \mathrm{~cm}$ holes. A houselight in the chamber's ceiling was illuminated at all times, except timeouts. Mixed grain was delivered by a food hopper centrally located below the viewing window.

\section{Stimuli}

Auditory stimuli were software-generated synthesized waveforms (Sonar version 4, Cakewalk, Boston). These 16-bit files were sampled at $44100 \mathrm{~Hz}$. The initial training stimuli were compound auditory stimuli. They were made from 12 pitch values from the third and fifth octaves (six from each octave, fundamental pitch range $=131-1046 \mathrm{~Hz}$ ) and 12 different timbres. The timbre of the 12 instruments were synthesized by combining the distinct harmonic and partial frequencies to the fundamental frequency of each pitch to simulate the harmonic spectral signature of each instrument (which also enlarged the total frequency spectrum by several $\mathrm{kHz}$ beyond the fundamental pitch depending on the instrument). These 12 pitch (labeled by note name and octave) and timbre values were uniquely combined to create 12 redundant auditory stimuli that were used during training (C3-piano, D\#3guitar, E3-vibraphone, G3-reed organ, G\#3-violin, B3harmonica, A5-alto Sax, A\#5-oboe D5-trumpet, F5-french horn, F\#5-flute, C6-clarinet). WAV files of these sounds were presented at $76-82 \mathrm{db}$ as measured from the bird's typical position in chamber (Radio Shack sound pressure meter; Weighting A).

\section{Go/No-Go Training}

Each trial started with a peck to a centrally located $2.5-\mathrm{cm}$ white circular warning signal. This was replaced by a $6.7-\mathrm{cm}$ purple square, to which the birds directed pecks during sound presentations. Same sound sequences consisted of a randomly selected training sound repeated 12 times. Different sound sequences consisted of single presentation of all 12 training sounds in a random- ized order that varied each trial. Each sequence lasted $18.55 \mathrm{~s}$, with each sound played for $1.5 \mathrm{~s}$ with a $50-\mathrm{ms}$ silent interstimulus interval (ISI) between them. Pecks to the purple stimulus during different trials $(\mathrm{S}+)$ were reinforced with $2.5 \mathrm{~s}$ access to grain on a variable interval (VI-8) schedule. During any within-trial reinforcements the sound sequences continued to be played. Different sequences were also reinforced at their termination (except for one bird in order to control weight). Pecks to same sequences $(\mathrm{S}-$ ) were never reinforced and resulted in a time-out that was proportional to the number of pecks emitted during the sequence. A small percentage of different sequences were tested as probe trials in which no reinforcement was permitted. These unreinforced probe trials allowed for the uncontaminated measurement of peck rate without the direct cues associated with reinforcement and the missed time for pecking the display that occurred while eating from the hopper. Only data from these probe trials were analyzed.

The pigeons were initially trained with sessions consisting of 100 randomly generated trials $(50 \mathrm{D} / 50 \mathrm{~S}$ trials $)$. After 60 sessions, only one pigeon showed evidence of limited learning. At this point, we used a modified training procedure. The pigeons continued to receive all possible sequences of different trials with all 12 stimuli, but the number of stimuli used to make the same trials was reduced and then gradually expanded as discriminative control was established. Under this new procedure, only one value was used to generate all same trials in a session at first. Once peck rates decreased for this sound sequence, a second sound was added to the pool of possible same sequences until both were mastered. The sounds tested on the same trials were randomly chosen from this growing pool of stimuli as it was gradually expanded over sessions. This procedure continued until all 12 sounds were reintroduced as same sequences, reestablishing a true S/D discrimination. ${ }^{1}$ During modified training, 60 trials $(30 \mathrm{D} / 30 \mathrm{~S}$ trials) were conducted each session, with eight randomly selected different sequences being unreinforced probe trials. Upon reestablishing the S/D discrimination, 16 steady-state sessions were conducted consisting of 48 different ( 8 probe trials) and 48 same trials. Generalized transfer testing then began.

\section{Novel Pitch/Timbre Combinations}

This transfer test examined novel recombinations of the 12 training pitches and timbres. Four sessions consisting of 96 baseline trials made from 12 training values (48 D/48 S trials) and 12 randomly inserted novel recombination trials (6 D/6 S trials) were conducted. The one constraint was that all transfer test trials occurred after the 30th trial of a session. For the transfer trials, the compound sounds tested novel recombinations of the training values and had not been heard before (e.g., A5-piano). Each new combination of values was tested only once. All transfer trials were

\footnotetext{
${ }^{1}$ It should be noted that we also started initially training two other birds with the S/D reinforcement contingencies reversed. Once we started the modified procedure, it become clear within two or three stimulus introductions that it was much easier for the birds to learn to inhibit pecking to new negative same sequences than to learn to peck vigorously at newly introduced positive same sequences. Because of their reputation for being difficult to train with auditory stimuli we thought it more important to establish an auditory S/D discrimination of any form to start, so we stopped training these two birds at this point.
} 
conducted as unreinforced probes. Because of a programming error in the interstimulus interval timing routine, a small percentage of these probe trials $(\approx 25 \%)$ were accidentally reinforced for a small proportion of pecks that occurred during the brief interstimulus intervals. This error was fixed and data from any contaminated transfer trials not analyzed.

\section{Dimensional Sensitivity Testing}

This transfer test examined two novel different conditions: pitch-only trials, in which the timbre of the instrument was held constant and only the pitch varied within a sequence and timbreonly trials in which the pitch was held constant and the timbre of the producing instrument was varied within a sequence. The 12 values tested with each dimension were the same as during training, although new combinations of pitch and timbre values were required to produce this dimensional specificity (i.e., the remaining 11 values not previously used in each dimension). Each session consisted of 96 baseline trials (48 D/48 S trials) and eight pitchonly trials (4 D/4 S trials) and eight timbre-only trials (4 D/4 S trials). In the first six sessions, the pitch-only trials were conducted using the French horn, and timbre-only trials used the F5 pitch. The next six sessions used the harmonica and B3 pitch. After testing, these two sets of pitch-only and timbre-only trials were added to their daily training. Following these test sessions, pitchonly and timbre-only trials using the same pitch and timbre values used during the test were incorporated into daily training sessions and the resulting same and different trials were differentially reinforced using the same contingencies as with the prior training values. Sessions now consisted of 80 trials, but with 16 redundant, 32 pitch-only, 32 timbre-only trials randomly intermixed in each session. Equal numbers of same and different sequences were tested with each type.

\section{Novel Timbres}

This transfer test examined sequences constructed of novel timbres. Six test sessions consisted of 80 baseline trials (16 redundant, 32 pitch-only, and 32 timbre-only trials) and eight novel timbre unreinforced probe trials $(4 \mathrm{D} / 4 \mathrm{~S})$ randomly intermixed. Using the F5 pitch, the sequences were randomly constructed from the "banjo," "clavinet," "bottle-blow," "brass," "celesta," "sitar," "contrabass," "dulcimer," "atmosphere," "fiddle," "shakuhachi," "sitar," and "voice" instruments available within the Sonar software package.

\section{Stimulus Set Expansion}

At this point, the entire set of possible redundant, pitch-only, and timbre-only sequences were introduced and added to daily testing. These trials involved all 144 possible sound combinations of our initial values ( 12 pitches $\times 12$ timbres) in making the same and different sequences. Just prior to this addition, we conducted three transfer sessions with novel nonreinforced pitch-only (“A\#5," "E3," "C3”) and timbre-only ("oboe," "vibraphone," "violin") trials similar to the earlier dimensional sensitivity test.

\section{Novel Pitches}

This transfer test examined sequences constructed from novel pitches from within and between the octaves used in training.
Stimuli for the within set were the 12 remaining untested pitches from the 3rd (6 notes) and 5th octaves (6 notes), while the between set were the notes from the fourth octave (12 pitches). Each session consisted of 80 baseline trials (16 redundant, 32 pitch-only and 32 timbre-only trials randomly selected from the 144 item pool) and 16 novel pitch transfer trials ( 8 within/ 8 between). These novel pitch values were carried by a familiar timbre (alto sax).

\section{Complex Sounds}

This transfer test examined sequences constructed from novel complex sounds. These consisted of six "natural" sounds ("dog bark," "bird chirp," "cat meow," "horse whinny," "lark call," "elephant trumpet") and "artificial" sounds ("bang," "horn," "light saber," "spring," "whip," "whistle"). Because the birds did not peck during these stimuli upon their introduction, these complex sounds were differentially trained for approximately two months before a second round of testing occurred. Thirty-two complex sound trials (16 D/16 S) were added to each training session using these stimuli, along with 16 pitch, 16 timbre, and 16 redundant trials. Following training, the pigeons were transfer tested with a second set of 12 novel complex sounds ("jackhammer," "piglet," "zip," “dolphin," "hyena," "snake," "blast," "cow," "party horn," "engine revving," "breaking glass," "wasp"). These 18 test sessions consisted of the 80 training trials and eight novel complex sound unreinforced probe trials (4 D/4 S).

\section{Results}

All three pigeons successfully learned the modified procedure and transferred this auditory S/D discrimination across a number of different dimensions and features of the stimuli. The primary measure used to evaluate this behavior was discrimination ratio, $(\mathrm{DR}=\mathrm{S}+$ pecks $/[\mathrm{S}+$ pecks $+\mathrm{S}-$ pecks $])$ across serial positions within a sequence. An alpha level of $p<.05$ was used to judge all statistical significance.

\section{Acquisition}

The pigeons initially showed some difficulty in learning the S/D task, with only pigeon $\# 2 \mathrm{~N}$ reliably above chance after 60 sessions. All the pigeons were subsequently successful at learning the incremental modification of the task. Table 1 shows the mean DR from the first session $(\mathrm{DR}=.59)$ with each new same sequence and the mean number of sessions required to incorporate it into the ongoing discrimination ( $\mathrm{X}=6.4$ sessions). Using just the $12 \mathrm{DR}$ scores from the first sessions, two pigeons were significantly above chance upon testing with the reintroduced stimuli, \#1N $t(11)=2.7 ; \# 2 \mathrm{~N}-t(11)=4.4$, and the third pigeon was close, $\# 3 \mathrm{~W}-t(11)=2.1, p=.06$.

\section{Steady State}

After the S/D task was reestablished, each bird was significantly above chance at discriminating same and different sound sequences. Over the first 16 sessions upon reestablishing the complete $\mathrm{S} / \mathrm{D}$ task, the mean $\mathrm{DR}$ for all birds was $.73(\# 1 \mathrm{~N}=.72$; $\# 2 \mathrm{~N}=.78 ; \# 3 \mathrm{~W}=.70)$. One advantage of this successive presentation procedure is the capacity to track the temporal trajectory of discrimination across a single trial (see Cook, Kelly, \& Katz, 
Table 1

Transfer and Sessions to Criterion for Successively Introduced New Sounds During Training

\begin{tabular}{|c|c|c|c|c|}
\hline & \multirow{2}{*}{$\frac{\text { Mean first session }}{\text { Discrimination ratio }}$} & \multicolumn{3}{|c|}{ Number of training sessions ${ }^{1}$} \\
\hline & & $\# 1 \mathrm{~N}$ & $\# 2 \mathrm{~N}$ & $\# 3 \mathrm{~W}$ \\
\hline Sound 1 (A5 - alto sax) & 70.5 & 2 & 2 & 2 \\
\hline Sound 2 (D5 - trumpet) & 55.7 & 6 & 5 & 5 \\
\hline Sound 3 (C6 - clarinet) & 67.4 & 3 & 2 & 11 \\
\hline Sound 4 (B3 - harmonica) & 56.5 & 6 & 6 & 10 \\
\hline Sound 5 (D\#3 - guitar) & 55.3 & 7 & 4 & 22 \\
\hline Sound 6 (E3 - vibraphone) & 56.5 & 10 & 3 & 7 \\
\hline Sound 7 (F\#5 - flute) & 54.7 & 10 & 4 & 20 \\
\hline Sound 8 (F5 - french horn) & 54.0 & 4 & 8 & 5 \\
\hline Sound 9 (G3 - reed organ) & 58.4 & 6 & 4 & 20 \\
\hline Sound $10(\mathrm{C} 3-$ piano $)$ & 54.5 & 11 & 5 & 17 \\
\hline Sound 11 (G\#3 - violin) & 57.2 & 2 & 5 & 3 \\
\hline Sound 12 (A\#5 - oboe) & 68.7 & 3 & 2 & 2 \\
\hline Mean & 59.1 & 5.8 & 3.2 & 10.3 \\
\hline
\end{tabular}

Note. $\quad{ }^{1}$ Number of sessions until two sessions within one standard deviation of baseline $\mathrm{S}-$ peck rate across acquisition.

2003). This analysis can reveal the microstructure of when and how the $\mathrm{S}+$ and $\mathrm{S}-$ sequences were discriminated. Figure 1 shows the mean DR based on the number of pecks made during each of the 12 sounds within a trial as collected during these 16 sessions. Across the sound serial positions, it shows that discrimination gradually and continually improved over the sequence, especially after the presentation of the third sound. A repeated measures ANOVA using polynomial contrasts confirmed a linear increase across trial serial position, $F(1,2)=401, \eta_{\mathrm{p}}^{2}=.99, p_{\text {rep }}=.98$. Comparing performance at each serial position with chance revealed that significant above-chance discriminations occurred by the fourth sound for one pigeon and by the fifth sound for the remaining two pigeons, $t \mathrm{~s}(15)>2.2$.

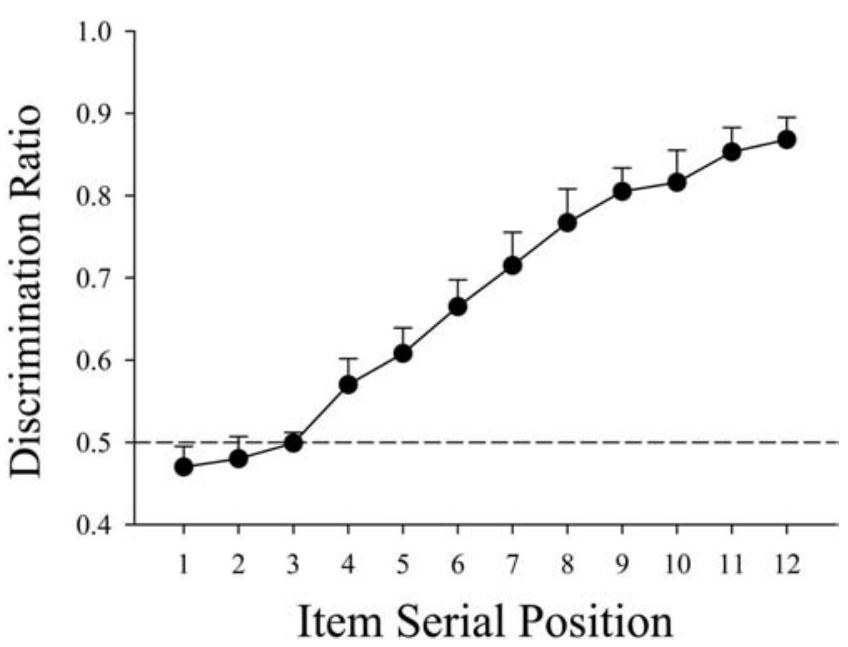

Figure 1. Mean discrimination ratio as determined by the number of pecks made during each of the 12 sounds across a trial's sequence. The dotted reference line depicts chance responding. Error bars show the SEM for each condition.

\section{Transfer}

All three pigeons transferred their auditory S/D discrimination across all six tests. Each panel of Figure 2 shows mean DR across 3-item groupings of these sequences for the transfer conditions and most comparable baseline comparison condition.

\section{Novel Pitch/Timbre Recombination}

Figure 2A shows DR for both the baseline and novel recombination probe trials. Baseline performance was slightly higher over latter segments of the sequence, but the transfer condition (.75) was significantly above-chance over the last six items, $t \mathrm{~s}(2)>5.9$. A repeated measures AVOVA (Session $\times$ Condition $\times$ Serial Position) confirmed this pattern, revealing a significant Condition $\times$ Serial Position interaction, $F(3,6)=10.3, \eta_{\mathrm{p}}^{2}=.84, p_{\text {rep }}=$ 95. Tests of just the novel transfer condition with polynominal contrasts confirmed the presence of a significant linear increase across serial position, $F(1,2)=55.8, \eta_{\mathrm{p}}^{2}=.96, p_{\text {rep }}=.93$. Although contaminated trials were removed from the analysis, we carefully examined the data for any training effect across sessions. No significant changes across testing were found.

\section{Dimensional Sensitivity}

Figure 2B shows DR for the baseline, novel pitch, and novel timbre-only sequences probe trials. Each dimensional condition supported above-chance transfer upon its separation from the redundant compound training stimuli. Pitch-only trials (.72) supporting better discrimination than did timbre-only trials (.67) over the last six items of the sequences. Over these items, both pitch-only and timbre-only conditions were significantly above chance, $t \mathrm{~s}(2)>5.5$. Both were significantly lower than with the baseline redundant condition. An AVOVA (Sessions $\times$ Condition $\times$ Serial Position) confirmed a significant linear effect of serial position, $F(1,2)=273, \eta_{\mathrm{p}}^{2}=.99, p_{\text {rep }}=.97$. and also hinted at a possible Condition $\times$ Serial Position interaction, $F(6,12)=2.5, p=.08$, $p_{\text {rep }}=.84$. Tests of only the transfer conditions revealed a signif- 

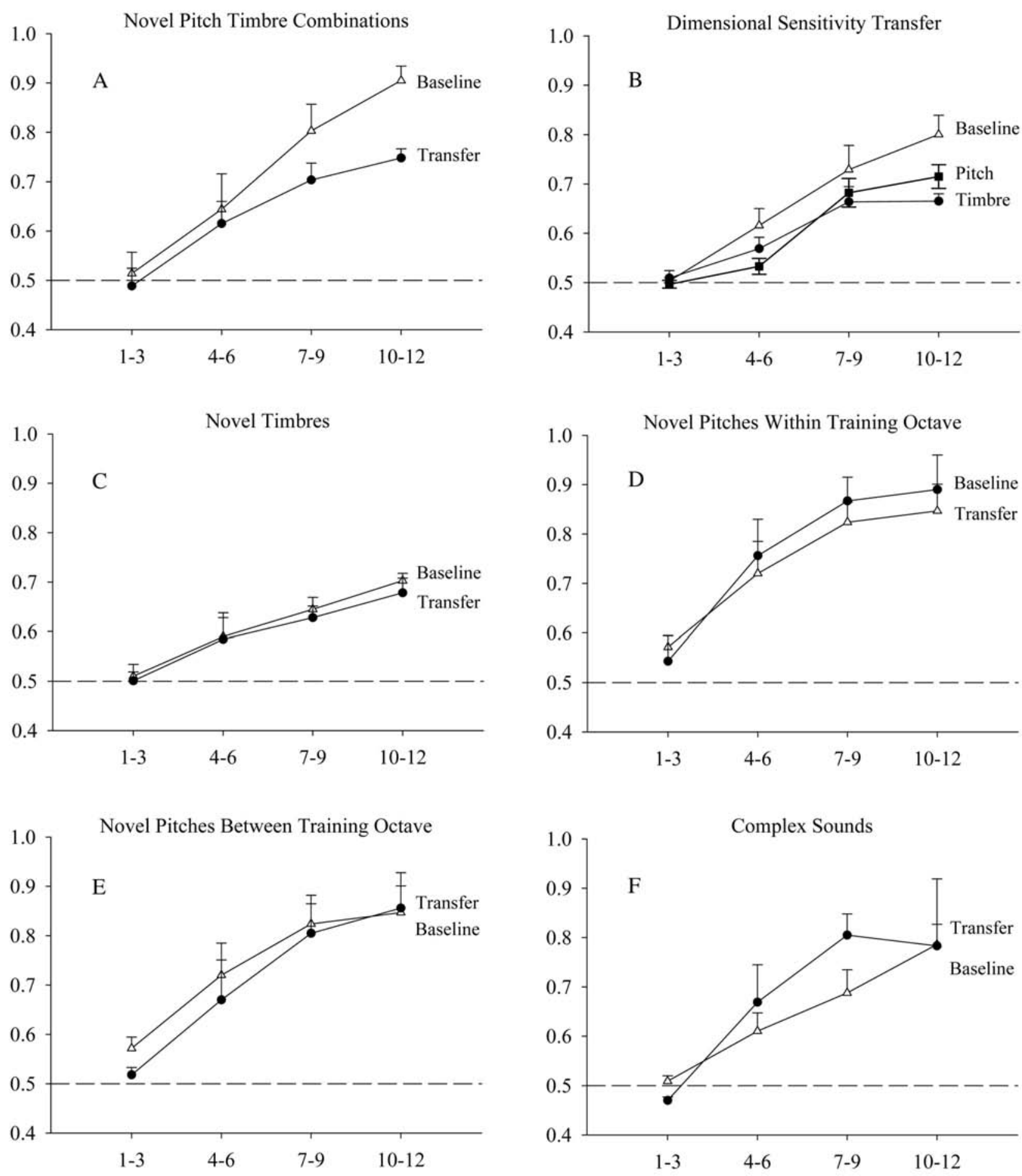

\section{Item Serial Position}

Figure 2. This figure shows the mean results from six different transfer conditions. Each panel shows discrimination ratio across grouping of three successive items within a presentation. The most appropriate baseline comparison is included for each test. The dotted reference lines depict chance responding. Error bars show the SEM for each condition. 
icant linear increases in DR across serial position for transfer pitch $F(1,2)=485.3, \eta_{\mathrm{p}}^{2}=.99, p_{\text {rep }}=.98$ and timbre conditions, $F(1$, $2)=22.4, \eta_{\mathrm{p}}^{2}=.92, p_{\text {rep }}=.89$. The results of the second transfer test of this type just prior to the increase in stimulus set size were indistinguishable from the first.

\section{Novel Timbres}

Figure 2C shows DR for the baseline and novel timbre probe trials. Discrimination improved for both conditions across serial position. An AVOVA revealed a significant effect of serial position, $F(3,6)=12.4, \eta_{\mathrm{p}}^{2}=.86, p_{\text {rep }}=.97$, but no main effect or interaction with condition. Over the last six novel items, transfer $\mathrm{DR}$ was significantly above chance, $t \mathrm{~s}(2)>5.4$. Tests of only the novel timbre condition revealed a significant linear increase of DR across serial position, $F(1,2)=34.4, \eta_{\mathrm{p}}^{2}=.95$, $p_{\text {rep }}=.91$.

\section{Within-Octave Test.}

Figure 2D shows DR for the baseline and novel pitch probe trials. Discrimination improved for both the baseline and transfer condition across serial position with the novel within-octave performance being equivalent to baseline. An AVOVA revealed a significant effect of Serial Position, $F(3,6)=21.8, \eta_{\mathrm{p}}^{2}=.92$, $p_{\text {rep }}=.98$, but no main effect or interaction with Condition. Over the last six novel items, transfer DR was significantly above chance, $t \mathrm{~s}(2)>5.1$. Tests of only the transfer pitch condition revealed a significant linear increase in DR across serial position, $F(1,2)=136.9 \eta_{\mathrm{p}}^{2}=.98 p_{\text {rep }}=.96$.

\section{Between-Octave Test}

Figure 2E shows DR for the baseline and novel between-octave pitch probe trials. Discrimination improved for both conditions across serial position An AVOVA revealed a significant effect of Serial Position, $F(3,6)=17.4, \eta_{\mathrm{p}}^{2}=.90, p_{\text {rep }}=.98$, and Condition, $F(1,2)=132.2, \eta_{\mathrm{p}}^{2}=.98, p_{\text {rep }}=.96$, as baseline discrimination was better than transfer performance. By the last three novel items, transfer DR was significantly above chance, $t(2)=$ 5.2 , and was close for items $6-9, t(2)=3.8, p=.06$. Tests of only the transfer pitch condition revealed a significant change of DR across serial position, $F(3,6)=6.4, \eta_{\mathrm{p}}^{2}=.76$, $p_{\text {rep }}=.91$.

\section{Complex Sounds}

Once the pigeons started to peck during the complex sounds, the pigeons showed significant improvement over discrimination training. Over the last 10 training sessions, each pigeon was significantly above chance over serial positions $9-12$, \#1N -.81 , $t(9)=9.6 ; \# 2 \mathrm{~N}-.62, t(9)=5.1 ; \# 3 \mathrm{~W}-.70, t(9)=5.4$. Figure $2 \mathrm{~F}$ shows DR for the baseline and the second set of novel complex novel probe trials. During this test there was no significant main effect or interaction with condition, but both conditions improved across serial position, $F(3,6)=4.8, \eta_{\mathrm{p}}^{2}=.71, p_{\text {rep }}=.88$. Bird \#2N was significantly above chance for serial positions 3-6 on the transfer trials, $t(5)=5.1$, while birds $\# 1 \mathrm{~N}$ and \#3W were significantly above chance, $t \mathrm{~s}(5)>4.4$ over the last nine novel items of the transfer sequence of complex sounds. Tests of only the complex sounds transfer condition revealed a significant quadratic trend for DR across serial position, $F(1,3)=39.9, \eta_{\mathrm{p}}^{2}=.95 .2$, $p_{\text {rep }}=.92$, as transfer performance apparently declined over the last three items.

\section{General Discussion}

These experiments show for the first time auditory S/D discrimination and transfer in pigeons. In six tests involving novel auditory sequences, the pigeons consistently exhibited significant discrimination transfer. These included tests with novel recombinations of training stimuli, separation into pitch and timbre-only conditions, novel pitches within and between the training octaves, novel instruments, and novel complex sounds. Although not detailed above, the discrimination was also well maintained when testing was expanded to include all possible combinations of the 144 pitch/timbre combinations. This generalized flexibility indicates that the previously demonstrated capacity to use and abstract S/D relations in pigeons is not limited to the visual modality, but extends to the auditory modality as well. Each presentation in the sequence of auditory stimuli improved discrimination indicating the involvement of an accumulative, memory-based, comparison process that can be used to recognize strings of same and different sounds that differ along the pitch and harmonic dimensions. In combination with previously reported visual results (Cook, Katz, \& Cavoto, 1997; Cook, Kelly, \& Katz, 2003), these new auditory results raise the possibility that this $\mathrm{S} / \mathrm{D}$ capacity is potentially due to a single, higher-order abstraction mechanism that can detect relations across different modalities (audition \& vision), types of information (pitch, timbre, color \& shape) and temporal arrangements (successive \& simultaneous).

Some mammals (dolphins and monkeys) have been previously demonstrated to learn S/D relations using either the auditory (Thompson \& Herman, 1977; Wright, Shyan, \& Jitsumori, 1990) or visual modalities (Mercado, Killebrew, Pack, Macha, \& Herman, 2000; Wright, Santiago, \& Sands, 1984). The current results indicate the same duality holds true for pigeons, despite the considerable phylogenetic distance between these animals. Although concept transfer was not specifically examined in their studies, Dooling and his colleagues have repeatedly taught budgerigars (Melopsittacus undulates) to make S/D discriminations using a wide variety of auditory differences including pitch and timbre (Dooling, Brown, Klump, \& Okanoya, 1992; Dooling, Brown, Park, Okanoya, \& Soli, 1987; Lohr \& Dooling, 1998; Okanoya \& Dooling, 1991; Park \& Dooling, 1985). The considerable number of field studies that have also documented numerous bird species capable of detecting changes in the songs of their territorial neighbors suggests that the capacity to detect sameness and difference may be a reasonably widespread capacity in birds. One interesting direction for future research will be to examine how the generalized nature of the current discrimination is mechanistically related to these simpler detections of change and stability in the environment.

One possible difference between the two modalities may be with regards to speed of detecting or processing S/D relations. The pigeons consistently needed several auditory presentations, approximately 3 to 5 , to consistently exhibit stimulus control within a sequence. We have previously found using successive visual presentations of color pictures clear evidence that S/D discrimination emerged after only the minimal number of two presentations (Cook, Kelly, \& Katz, 2003). Another difference between the 
modalities was that we needed an asymmetrical learning procedure for the auditory stimuli that we have not had to employ in our visual studies. At the moment, we are unsure of the reason for this latter difference. It is not even clear if this procedural variation was required had we just exhibited more patience. Nevertheless, we have found each of our visual S/D discriminations easier to train, at least on the surface. One key difference between the modalities that may have been critical in both of the above differences is that in visual settings the pigeons can peck directly at the critical stimuli, in a way not possible with the less tangible auditory stimuli. This difference in the directness of the observing response is certainly a factor that merits further examination. Bearing these differences in mind, there is little reason to believe pigeons are profoundly different in their capacity to carry out S/D discriminations between modalities.

What was the acoustic basis for the discrimination? One possibility we considered was the simple detection of pitch transitions or immediate changes in the perceptual flux between temporally adjacent items. Accounts relying exclusively on the simple detection of low-level perceptual transients have not been supported in our visual work (Cook \& Blaisdell, 2006). While such short lived factors may contribute, it is certainly not the critical basis for the S/D discrimination in the present study. This is indicated by the success that the pigeons had with discriminating between the timbres of different instruments and between the complex sounds. The pitch contour and spectral content of these sounds were irregular, requiring an extended temporal segment of each sound to be compared in memory to the previous items to determine the relative similarity among current and past items. Furthermore, in observations not included here, we found that the pigeons could easily tolerate delays of two seconds between successive auditory items in a sequence also indicating that a working memory for the sequence of items is involved. Such results, combined with cumulative within-trial progress of the discrimination, indicate that auditory short-term memories lasting over many seconds play an important role in this discrimination. Given the tonal nature of our stimuli, the fundamental frequency of each pitch was likely an important cue, but the entire harmonic spectrum must also have been involved in the discrimination of the different instrumental timbres and complex sounds. Finally, it should be noted that the first set of complex stimuli did need a short period of retraining before the pigeons subsequently showed successful transfer in the second test. The very low rate of pecking upon their initial introduction likely reflects the recognition of the considerable difference between these kinds of sounds and those used during initial training. The instrumental notes used during training often sustained for the entire period of presentation, whereas the complex sounds had far more complex harmonic structures that ebbed and flowed over time. Thus, the birds may have had a S/D rule at their introduction, but it was limited to auditory values that were reasonably similar to such extended notes (i.e., transfer Test 1-5). Once the birds had experienced these more complex stimuli, they appear to have easily extended it to encompass a wider variety of auditory experience.

Despite the relational control exhibited above, the contribution and interaction between relational and absolute factors in auditory and visual stimulus control is still an unresolved issue in birds.
Within the visual domain, birds have been shown to have the propensity of being controlled by both absolute and relational factors (Cook, Kelly, \& Katz, 2003; Cook, Levison, Gillett, \& Blaisdell, 2005; Wasserman, Frank, \& Young, 2002). The same appears true for the auditory modality. Within the auditory modality, reports have indicated that some birds can distinguish among relatively large numbers (>64) of conspecific songs (Chew, Vicario, \& Notebohm, 1996; Stoddard, Beecher, Loesche, \& Campbell, 1992) and mockingbirds have learned repertoires of 150 or more heterospecific songs (Derrickson, 1987) suggesting good absolute stimulus control. Starlings, zebra finches, and pigeons have been shown to be constrained in their transfer across rising and falling pitch sequences by the memorization of the absolute training pitches (Cynx, 1995; Page, Hulse, \& Cynx, 1989). Further, it has been found in discriminations that require memorization of absolute pitch that several bird species, including pigeons, are better than mammals (Friedrich, Zentall, \& Weisman, 2007; Weisman, Njegovan, Williams, Cohen, \& Sturdy, 2004). Based on such results, it has recently suggested that birds in general are more attuned to the feature of absolute pitch than mammals (Weisman, Njegovan, Williams, Cohen, \& Sturdy, 2004). Persistent attempts to encode such absolute factors in the present stimuli may have been one additional reason why the relational control in the present task seemed more difficult to generate than in the visual domain. Research on how these competing factors come to control behavior in any discrimination and how they are influenced by task considerations, modality, and phylogenetic considerations are clearly needed.

Finally, another key question is whether this newly established relational auditory control and previous visual control indicates the operation of two separate modality-specific mechanisms or one generalized, higher-order, mechanism that can abstract such information independent of modality. At the moment, this important theoretical issue cannot be resolved. While the overt similarity between the present auditory S/D results and prior visual S/D results suggests a possible common mechanism, results from other procedures have suggested that stimulation in the two modalities can produce different effects (Delius \& Emmerton, 1978; Foree \& Lolordo, 1973; Kelley, 1986; Santi, Stanford, \& Coyle, 1998). The latter findings suggest potentially separate and modality-specific learning mechanisms. An important start on addressing this critical issue will be to look for cross modal transfer in the learning of S/D relations between modalities. If learning in one modality can be shown to influence behavior in the other, it would indicate a higher level of abstractness to pigeon S/D behavior, something already suggested by their flexible solutions applied within the visual domain (Cook \& Wasserman, 2006).

The ability to compare the identity and differences across auditory streams of information not only is important to adaptively integrating behavior across time, but is a critical component to the emergence and evolution of acoustic communication, and eventually language and music. The current results indicate that the capacity to detect and abstract sequential sameness and difference can be found across multiple modalities. Intriguingly, the next step of attaching meanings to these acoustic streams of information for the purposes of communication may also be within the capacity of some birds (Templeton, Greene, \& Davis, 2005). 


\section{References}

Chew, S. J., Vicario, D. S., \& Notebohm, F. (1996). A large-capacity memory system that recognizes the calls and songs of individual birds. Proceedings of the National Academy of Sciences, USA, 93, 1950-1955.

Cook, R. G., \& Blaisdell, A. P. (2006). Item memory in successive same-different discriminations. Behavioural Processes, 72, 255-264.

Cook, R. G., Katz, J. S., \& Cavoto, B. R. (1997). Pigeon same-different concept learning with multiple stimulus classes. Journal of Experimental Psychology: Animal Behavior Processes, 23, 417-433.

Cook, R. G., Kelly, D. M., \& Katz, J. S. (2003). Successive two-item same-different discrimination and concept learning by pigeons. Behavioural Processes, 62, 125-144.

Cook, R. G., Levison, D. G., Gillett, S. R., \& Blaisdell, A. P. (2005). Capacity and limits of associative memory in pigeons. Psychonomic Bulletin \& Review, 12, 350-358.

Cook, R. G., \& Wasserman, E. A. (2006). Relational learning in pigeons. In E. A. Wasserman \& T. Zentall (Eds.), Comparative cognition: Experimental explorations of animal intelligence (pp. 307-324). London, UK: Oxford University Press.

Cynx, J. (1995). Similarities in absolute and relative pitch perception in song birds (starling and zebra finch) and a non-song bird (pigeon). Journal of Comparative Psychology, 109, 261-267.

Delius, J. D., \& Emmerton, J. (1978). Stimulus-dependent asymmetry in classical and instrumental discrimination learning by pigeons. Psychological Record, 28, 425-434.

Derrickson, K. C. (1987). Yearly and situational changes in the estimate of repertoire size in Northern Mockingbirds (Mimus polyglottos). Auk, 104, 198-207.

Dooling, R. J., Brown, S. D., Klump, G., \& Okanoya, K. (1992). Auditory perception of conspecific and heterospecific vocalizations in birds: Evidence for special processes. Journal of Comparative Psychology, 106, $20-28$.

Dooling, R. J., Brown, S. D., Park, T. J., Okanoya, K., \& Soli, S. D. (1987). Perceptual organization of acoustic stimuli by budgerigars (Melopsittacus undulatus): I. Pure tones. Journal of Comparative Psychology, 101, 139-149.

Foree, D. D., \& Lolordo, V. M. (1973). Attention in the pigeon: Differential effects of food-getting versus shock-avoidance procedures. Journal of Comparative \& Physiological Psychology, 85, 551-558.

Friedrich, A., Zentall, T. R., \& Weisman, R. G. (2007). Absolute pitch: Frequency-range discriminations in pigeons (Columba livia)Comparisons with zebra finches (Taeniopygia guttata) and humans (Homo sapiens). Journal of Comparative Psychology, 121, 95-105.

Harrison, J. B., \& Furumoto, L. (1971). Pigeon audiograms: Comparison of evoked potential and behavioral thresholds in individual birds. Journal of Auditory Research, 11, 33-42.

Heise, G. A. (1953). Auditory thresholds in the pigeon. American Journal of Psychology, 66, 1-19.

Jenkins, H. M., \& Harrison, R. H. (1960). Effect of discrimination training on auditory generalization. Journal of Experimental Psychology, 59, $246-253$.

Katz, J. S., \& Wright, A. A. (2006). Same/different abstract-concept learning by pigeons. Journal of Experimental Psychology: Animal Behavior Processes, 32, 80-86.

Kelley, M. J. (1986). Selective attention and stimulus-reinforcer interactions in the pigeon. Quarterly Journal of Experimental Psychology: Comparative \& Physiological Psychology, 38B, 97-110.

Kraemer, P. J., \& Roberts, W. A. (1985). Short-term memory for simultaneously presented visual and auditory siginals in the pigeon. Journal of Experimental Psychology: Animal Behavior Processes, 11, 137-151.

Lohr, B., \& Dooling, R. J. (1998). Detection of changes in timbre and harmonicity in complex sounds by zebra finches (Taeniopygia guttata) and budgerigars (Melopsittacus undulatus). Journal of Comparative Psychology, 112, 36-47.
Mackintosh, N. J. (2000). Abstraction and discrimination. In C. Heyes \& L. Huber (Eds.), The evolution of cognition (pp. 123-142). Cambridge, MA: MIT Press.

Mercado, E., III, Killebrew, D. A., Pack, A. A., Macha, I. V. B., \& Herman, L. M. (2000). Generalization of 'same-different' classification abilities in bottlenosed dolphins. Behavioural Processes, 50, 79-94.

Okanoya, K., \& Dooling, R. J. (1991). Perception of distance calls by buderigars (Melopsittacus undulatus) and zebra finches (Poephilia guttata): Assessing species-specific advantages. Journal of Comparative Psychology, 105, 60-72.

Page, S. C., Hulse, S. H., \& Cynx, J. (1989). Relative pitch perception in the European starling (Sturnus vulgaris): Further evidence for an elusive phenomenon. Journal of Experimental Psychology: Animal Behavior Processes, 15, 137-146.

Park, T. J., \& Dooling, R. J. (1985). Perception of species-specific contact calls by the budgerigar (Melopsittacus undulatus). Journal of Comparative Psychology, 99, 391-402.

Pepperberg, I. M. (1987). Acquisition of the same/different concept by an African Grey parrot (Psittacus erithacus): Learning with respect to categories of color, shape, and material. Animal Learning \& Behavior, $15,423-432$.

Santi, A., Stanford, L., \& Coyle, J. (1998). Pigeons' memory for event duration: Differences between visual and auditory signals. Animal Learning \& Behavior, 26, 163-171.

Sinnott, J. M., Sachs, M. B., \& Hienz, R. D. (1980). Aspects of frequency discrimination in passerine birds and pigeons. Journal of Comparative \& Physiological Psychology, 94, 401-415.

Skutch, A. F. (1991). Life of the Pigeon. Ithaca, NY: Comstack Publishing. Stoddard, P. K., Beecher, M. D., Loesche, P., \& Campbell, S. E. (1992). Memory does not constrain individual recognition in a bird with song repertoires. Behaviour, 122, 274-287.

Templeton, C. N., Greene, E., \& Davis, K. (2005). Allometry of alarm calls: Black-capped chickadees encode information about predator size. Science, 308, 1934-1937.

Thompson, R. K. R., \& Herman, L. M. (1977). Memory for lists of sounds by the bottle-nosed dolphin: Convergence of memory processes with humans? Science, 195, 501-503.

Thompson, R. K. R., \& Oden, D. L. (2000). Categorical perception and conceptual judgments by nonhuman primates: The paleological monkey and the analogical ape. Cognitive Science, 24, 363-396.

Wasserman, E. A., Frank, A. J., \& Young, M. E. (2002). Stimulus control by same-versus-different relations among multiple visual stimuli. Journal of Experimental Psychology: Animal Behavior Processes, 28, 347-357.

Weisman, R. G., Njegovan, M. G., Williams, M. T., Cohen, J. S., \& Sturdy, C. B. (2004). A behavior analyses of absolute pitch: Sex, experience and species. Behavioural Processes, 66, 289-307.

Wright, A. A., Cook, R. G., \& Kendrick, D. F. (1989). Relational and absolute stimulus learning by monkeys in a memory task. Journal of the Experimental Analysis of Behavior, 52, 237-248.

Wright, A. A., Santiago, H. C., \& Sands, S. F. (1984). Monkey memory: Same/different concept learning, serial probe acquisition, and probe delay effects. Journal of Experimental Psychology: Animal Behavior Processes, 10, 513-529.

Wright, A. A., Shyan, M. R., \& Jitsumori, M. (1990). Auditory same/ different concept learning by monkeys. Animal Learning \& Behavior, $18,287-294$.

Young, M. E., \& Wasserman, E. A. (2001). Evidence for a conceptual account of same-different discrimination learning in the pigeon. Psychonomic Bulletin \& Review, 8, 677-684.

Received October 21, 2005

Revision received July 6, 2007

Accepted July 10, 2007 